\title{
Increasing fast food portion sizes: myth or reality?
}

\author{
R. Doody ${ }^{1}$, W. L. Wrieden ${ }^{1}$ and K. L. Barton ${ }^{2}$ \\ ${ }^{1}$ School of Pharmacy and Life Sciences, Robert Gordon University, Aberdeen, AB25 1HG, UK and ${ }^{2}$ Centre for Public \\ Health Nutrition Research, University of Dundee, Dundee DDI 9SY, UK
}

Evidence from the USA suggests that portion sizes for fast foods such as soft drinks, hamburgers and French fries, have increased over the last 20 years ${ }^{(1,2)}$. There is some evidence of increasing portion sizes in fast-food outlets in the UK, but the increases in energy intakes resulting from this change are not as large as those reported in the USA ${ }^{(3,4)}$

The aim of this work was to compare portion sizes, of foods from fast food chains, collected in 2004/2005 ${ }^{(5)}$ with portion sizes of equivalent products from 2010. Portion size data of 352 different products given on the websites of five popular fast food outlets in March 2010 was compared to that collected in 2004/2005. Mean portion sizes from 2004/2005 and 2010 were compared using paired $t$-tests after grouping into categories by food type and manufacturer, for example the mean of McDonalds burger weights from 2010 was compared with corresponding mean of McDonalds burgers from 2004/2005. A selection of results, are presented in the table.

\begin{tabular}{|c|c|c|c|c|c|c|c|}
\hline \multirow[b]{2}{*}{ Product } & \multirow[b]{2}{*}{ Manufacturer } & \multicolumn{2}{|c|}{$2004 / 2005$} & \multicolumn{2}{|c|}{2010} & \multirow[b]{2}{*}{$n$} & \multirow[b]{2}{*}{$P$} \\
\hline & & $\overline{\text { Mean }(\mathrm{g})}$ & $\overline{\mathrm{SD}}$ & $\overline{\text { Mean }(\mathrm{g})}$ & $\overline{\mathrm{SD}}$ & & \\
\hline Beef Burgers, standard & Burger King & 153 & 26 & 146 & 24.4 & 6 & 0.002 \\
\hline Beef Burgers, large & Burger King & 322 & 44 & 322 & 44 & 5 & $\mathrm{n} / \mathrm{a}$ \\
\hline Beef Burgers, average & McDonalds & 166 & 45 & 173 & 53 & 6 & 0.561 \\
\hline \multirow[t]{2}{*}{ Pizza slice, small } & Dominos & 66 & 10 & 74 & 8 & 20 & 0.002 \\
\hline & Pizza Hut & 80 & 6 & 82 & 9 & 10 & 0.377 \\
\hline \multirow{2}{*}{ Pizza slice, medium } & Dominos & 78 & 10 & 83 & 9 & 21 & 0.010 \\
\hline & Pizza Hut & 111 & 9 & 112 & 10 & 10 & 0.864 \\
\hline \multirow[t]{2}{*}{ Pizza slice, large } & Dominos & 85 & 13 & 89 & 9 & 20 & 0.032 \\
\hline & Pizza Hut & 129 & 12 & 123 & 15 & 10 & 0.154 \\
\hline Sandwiches, low fat & Subway & 241 & 31 & 228 & 33 & 8 & $<0.001$ \\
\hline Sandwiches, hot & Subway & 268 & 22 & 262 & 30 & 8 & 0.229 \\
\hline
\end{tabular}

There were few significant changes in portion weight over the period, and for the products evaluated there were as many decreases as increases in size. There was some evidence to suggest fast food portion sizes are increasing in the UK but the trend was restricted to one pizza chain. Decreases in portion sizes of sandwiches were noted at one chain. The introduction of smaller portion sizes and the discontinuation of the largest portion sizes was also observed. Increases in portion sizes were not always accompanied by portion size name changes, for example large hash browns in 2004/2005 are now $28 \mathrm{~g}$ heavier, yet the portion size is still described as large. Overall the observation was that there was a greater range of portion sizes than was previously seen, ensuring that consumers have more choice and that smaller portion sizes are available if desired. However, marketplace sizes remain excessive and do not reflect consumers' daily energy needs.

In conclusion, there is no firm evidence that portion sizes of burgers, pizzas and sandwiches available from the most popular fast food outlets have increased in size over the period 2004/2005 to 2010 but the introduction of new products, some with an increased energy density (e.g. stuffed crust pizza), cannot rule out an increase in energy intake from such products.

1. Young LR \& Nestle M (2002) Am J Public Health 92, 246-249.

2. Nielsen SJ \& Popkin BM (2003) JAMA 289, 450-453.

3. Wrieden WL, Gregor A \& Barton KL (2008) Proc Nut Soc 67, OCE6, E211.

4. Church S (2008) Trends in Portion Sizes in the UK - A Preliminary Review of Publishes Information. London: Food Standards Agency.

5. Wrieden WL \& Barton KL (2005) Calculation and Collation of Typical Food Portion Sizes for Adults aged $19-64$ and Older People Aged 65 and over. (N08026). London: Food Standards Agency. 